\title{
Welcome to the year 2018
}

\author{
Nadia Magnenat-Thalmann ${ }^{1}$
}

(C) Springer-Verlag GmbH Germany, part of Springer Nature 2017

The Visual Computer Journal is progressing extremely well. The impact factor has increased to 1.468, and we have received more than 592 submissions during the year. Our acceptance rate is about $23 \%$. Each submitted paper is handled by an Associate Editor (AE) that finds at least three reviewers to review the paper. I take the opportunity to thank all of them for their tremendous and invaluable work.

Throughout 2017, some Associate Editors have left the editorial board of the Visual Computer. I would like to thank them for their great work and contribution.

Here is the list of the Associate Editors who have left the editorial board:

- Kadi Bouatouch, IRISA, University of Rennes I, France.

- Gang Hua, Microsoft Asia, China.

- Yasuyuki Matsushita, Osaka University, Japan.

- Jacob Munkberg, Intel Corporation, Sweden.

I would like to welcome the new Associate Editors who have joined the editorial board during 2017:

- Jianfei Cai, Nanyang Technological University, Singapore.

- Yiyu Cai, Nanyang Technological University, Singapore.

- Jie Chen, University of Oulu, Finland.

- David Coeurjolly, CNRS, LIRIS, University of Lyon, France.

- Jean-Michel Dischler, University of Strasbourg, France.

- Parris K. Egbert, Brigham Young University, USA.

Nadia Magnenat-Thalmann

thalmann@miralab.ch; nadiathalmann@ntu.edu.sg;

visualcomputer@miralab.ch

1 MIRALab-CUI, University of Geneva, Battelle, Building A, 7, Route de Drize, Carouge, 1227 Geneva, Switzerland
- Tal Hassner, University of Southern California, USA.

- Shahar Kovalsky, Duke University, USA.

- Arjan Kuijper, Fraunhofer IGD \& TU Darmstadt, Germany.

- Risheng Liu, Dalian University of Technology, China.

- Xiaoyong Mao, University of Yamanashi, Japan.

- Jingjing Meng, Nanyang Technological University, Singapore.

- Beatriz Remeseiro, University of Oviedo, Spain.

- Leonid Sigal, University of British Columbia, Canada.

- Alexei Sourin, Nanyang Technological University, Singapore.

- Didier Stricker, Technical University Kaiserslautern and DFKI, Germany.

- Jinhui Tang, Nanjing University of Science and Technology, China.

- Wenming Zheng, Southeast University, China.

The editorial board of the Visual Computer is renewed regularly, and we welcome our new Associate Editors.

This year, we have introduced new topics and new keywords for the Visual Computer: We expect more papers on machine learning for graphics, pattern recognition, robotics and vision. We invite the research community to propose special issues in these topics.

During 2017, the journal published 35 best papers of the Computer Graphics Conference (CGI'2017) that was held in Japan in June 2017. We have also published best papers from other conferences such as VS Games 2015, ICVRV 2015, 3DOR 2015 and Cyberworlds 2016.

I would like to thank the Editorial Assistant, Juzheng Zhang, for his collaboration in the management of the journal over the year 2017 and welcome to the new Editorial Assistants Manoj Ramanathan and Nisha Jain. 
Finally, this journal could not exist without the strong support of our Editorial Director at Springer, Beverley Ford, as well as Rachel Roberts, the Editor of the journal. Thank you also to Beate Uhl, the Production Editor, and also to Vidhya Velayudhan for their continuous work and great assistance. Lastly, thank you to all the readers of the Visual
Computer who are a key factor to the success of the journal.

Nadia Magnenat-Thalmann

Editor-in-chief

The Visual Computer 